\title{
Energy Management Strategy Design for Series Hybrid Electric Vehicles
}

\author{
Iman Shafikhani
}





\title{
Energy Management Strategy Design for Series Hybrid Electric Vehicles
}

\author{
Iman Shafikhani
}

Linköping University

Department of Electrical Engineering

Division of Vehicular Systems

SE-581 83 Linköping, Sweden

Linköping 2021 
(c) EY-NC This work is licensed under a Creative Commons AttributionNonCommercial 4.0 International License.

https://creativecommons.org/licenses/by-nc/4.0/

Edition 1:1

(C) Iman Shafikhani, 2021

ISBN 978-91-7929-016-0

ISSN 0345-7524

URL

http://urn.kb. se/resolve?urn=urn:nbn:se:liu:diva-178628

Published articles have been reprinted with permission from the respective copyright holder.

Typeset using IATEX $_{\mathrm{E}}$

Printed by LiU-Tryck, Linköping 2021 
Here with a Loaf of Bread beneath the Bough,

A Flask of Wine, a Book of Verseand Thou Beside me

singing in the Wilderness-

And Wilderness is Paradise enow.

Omar Khayyam 

To My Parents 



\section{POPULÄRVETENSKAPLIG SAMMANFATTNING}

Elektrifiering av fordon är ett viktigt steg för att förbättra bränsleekonomi och reducera utsläpp från fossila drivmedel och marknaden för hybridfordon har ökat i popularitet som ett steg i denna riktning. Med en ökande omtanke om miljön har behovet av utveckling av relevant teknologi blivit mer märkbar än tidigare. Design av effektiva strategier för energistyrning som hanterar effektfördelningen mellan olika energikällor är en viktig del i denna strävan.

Denna avhandling inriktar sig på design av system för energihantering i seriehybrider, framförallt hybrid-elektriska hjullastare. I den första delen av arbetet tas hänsyn till batteriets livslängd när bränsleeffektiva strategier utvecklas. Detta är en viktig aspekt, eftersom kostnaden för batterier är en stor del av fordonets totala kostnad. En tidigare utvecklad empirisk modell för batteriets degradering modifieras och används för att förutsäga batteriets livslängd och i optimeringsproblemen som studeras vägs bränsleförbrukning och batteridegradering ihop. Två optimeringsramverk används för att analysera problemet, nämligen Pontryagins minimumprincip (PMP) och modellprediktiv reglering.

Simuleringar visar att det kan finnas en stor möjlighet att förlänga batteriets livslängd avsevärt med en liten ökning i bränsleförbrukning. När PMP-ramverket används har den utvecklade metodiken en prestanda med negativ korrelation med det kvadratiska medelvärdet av effektbehovet för de olika körcyklerna. Resultaten kan användas för att utveckla regelbaserade metoder som kan användas i realtid. I den andra delen av avhandlingen utvecklas effektiva optimeringsalgoritmer för elektriska seriehybrider och syftet är att uppnå enkla och effektiva reglerstrategier. 


\begin{abstract}
Electrification of vehicles is an indispensable step in improving fuel economy and reducing fossil fuel emissions. In particular, hybrid electric vehicle market has gained popularity as one such reliable solution. With the global rise in environmental concerns, the need for advancement of the relevant technologies has become more noticeable than before. In this pursuit, it is well-known that design of effective energy management strategy (EMS) that governs power distribution among the onboard energy sources is key in reducing fuel consumption and its adverse environmental impacts. This thesis is concerned with EMS design for series hybrid electric vehicles from two standpoints.
\end{abstract}

Powertrain component durability is often neglected in EMS development. In particular, batteries are prone to degradation through usage, a phenomenon widely known as cycle aging, and contribute largely to vehicle cost. In the first part of the thesis, therefore, battery lifetime optimization is integrated into the design of fuel-efficient energy management strategies. An empirical capacity degradation model is adopted from the literature and is modified in order to predict battery lifetime. The multi-objective problem is to compromise between fuel consumption reduction and battery wear minimization. The problem is formulated within two control theory frameworks, namely Pontryagin's minimum principle and model predictive control. Simulation results suggest that there is a remarkable potential in prolonging battery lifetime by sacrificing negligible to no excessive amount of fuel consumption. The application considered in this part is a hybrid electric wheel loader. While prolonging battery lifetime is economically beneficial for any hybrid electric vehicle, the cost savings for high power applications such as the aforementioned construction equipment can be even more rewarding.

The second part of the thesis is dedicated to development of time-efficient energy management strategies. Considering the need for real-time feasibility, satisfactory fuel economy and low computation time are the key elements in EMS design. In the first step, an analytical solution to the equivalent consumption minimization strategy (ECMS) for series hybrid electric vehicles is derived, where the system constraints are directly taken into account in the derivation process. Effective equivalence factor bounds are found and used to develop a real time adaptive ECMS. The obtained fuel economy figures are observed to be very close to the non-causal benchmarks. In the second step, the analytical policies are utilized to propose realtime predictive ECMS algorithms. Two scenarios are investigated depending on availability of drive cycle knowledge. The first scenario corresponds to vehicles that are expected to fulfill predefined tasks. This situation is common among construction machinery such as the wheel loader under study. In the second scenario, prior knowledge on driving mission is not accessible, and driver behavior is difficult to predict. This is a typical scenario encountered in urban driving. For each scenario, an algorithm is presented to compute the equivalence factor efficiently in a receding horizon fashion, and control action is determined by the analytical policy derived previously. Simulations of the developed algorithms for the hybrid wheel loader and a passenger car demonstrate that the methodologies are computationally efficient and attain satisfactory fuel economy with respect to dynamic programming benchmarks. 


\section{Acknowledgments}

I would like to express my gratitude to my main supervisor, Jan Åslund, for guidance, co-authorship, and encouragement. I am thankful to my co-supervisors, Erik Frisk and Christofer Sundström, for their co-authorship and valuable inputs. Erik is further acknowledged for prompt email responses and always being of help despite his busy schedule. I appreciate our colleagues at Volvo Construction Equipment, namely Anders Fröberg, Alessandro Carnevali, Elfving Auayporn, and Magnus Larsson, for technical support and hospitality. Jens Groot from Volvo Group Trucks Technology is thanked for providing us with battery data. The staff at the vehicular systems group are thanked for providing a friendly environment. Lars Eriksson is acknowledged for the teaching opportunity in vehicle propulsion systems course, from which I learned a lot.

Special thanks to Mahdi Morsali for intriguing discussions, long strolls, and the projects we worked on together. I am blessed to have a dependable friend like you. Shervin Parvini Ahmadi from the automatic control group is acknowledged for his genuine friendship and the nostalgic memories we made ever since coming to Sweden. Majid Moeinifard, Viktor Skyrman and Milad Moghaddassi are thanked for unforgettable memories that kept me sane during these years.

This work is dedicated to my beloved parents for their never-ending encouragements, support, and sacrifices. I appreciate my siblings for encouraging me along the way as well. As the list is long, I take this opportunity to acknowledge anyone who positively contributed to my $\mathrm{PhD}$ life and made it more colorful.

\section{Funding}

Swedish Energy Agency (Energimyndigheten) is acknowledged for funding the research. 



\section{Contents}

Abstract vii

$\begin{array}{ll}\text { Acknowledgments } & \text { ix }\end{array}$

Contents

1 Introduction 1

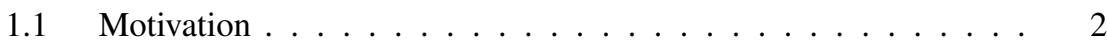

2 Background $\mathbf{5}$

2.1 Energy management strategies . . . . . . . . . . . . 6

2.2 Summary and contributions . . . . . . . . . . . . . . . 15

2.3 Reflection and future research outlook . . . . . . . . . 20

$\begin{array}{ll}\text { Bibliography } & 23\end{array}$

3 Paper I 33

3.1 Introduction . . . . . . . . . . . . . . . . 34

3.2 Vehicle model description . . . . . . . . . . . . . 36

3.3 Energy management problem formulation . . . . . . . . . . . . 48

3.4 Solution to the optimal control Problem . . . . . . . . . . . . . 51

3.5 Implementation of optimal controller for hybrid electric wheel loader 52

3.6 Case Study . . . . . . . . . . . . . . . . . . 55

3.7 Conclusion and Future Work . . . . . . . . . . . . . 63

References ..................... 65

4 Paper II $\quad \mathbf{7 1}$

4.1 Introduction . . . . . . . . . . . . . . . . . 72

4.2 Vehicle description .................. 72

4.3 Energy management problem formulation and design . . . . . . 76

4.4 Simulation . . . . . . . . . . . . . . . . . 78

4.5 Conclusions ....................... 83 
References ....................... 85

$5 \quad$ Paper III $\quad 89$

5.1 Introduction . . . . . . . . . . . . . . 93

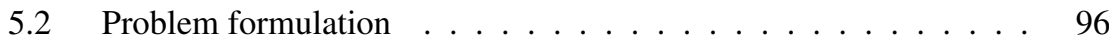

5.3 Analytical solution . . . . . . . . . . . . . 101

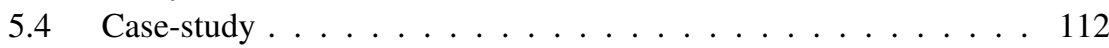

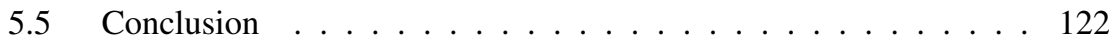

References ....................... 125

6 Paper IV 131

6.1 Introduction . . . . . . . . . . . . . . . 136

6.2 System model . . . . . . . . . . . . . . . . . . . 139

6.3 Equivalent Consumption Minimization Strategy . . . . . . . . . . 142

6.4 Predictive ECMS . . . . . . . . . . . . . . . . . . . . 146

6.5 Simulation results and discussion . . . . . . . . . . . . . 159

6.6 Conclusion and future work . . . . . . . . . . . . . 165

References ......................... 172 


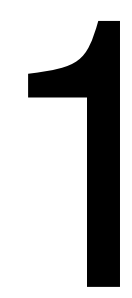

\title{
INTRODUCTION
}

\author{
Freedom from the desire for an \\ answer is essential to the \\ understanding of the problem.
}

J. Krishnamurti

Electric hybridization is a viable approach to reduce fuel consumption and environmental impacts of conventional vehicles, and as a result, hybrid electric vehicle (HEV) sector has gained lots of attraction among researchers of diverse backgrounds. The advantages of HEVs over conventional vehicles include downsizing of engine to reduce friction losses, recuperation of energy during braking phases to use later in order to save fuel, reduction in fuel consumption and emissions by cutting unnecessary idling losses via engine shut-off mechanism, and using engine in high-efficiency regions by avoiding part-load operation (Huang et al., 2017).

Performance of an HEV is sensitive to power flow through its powertrain components. Regarding fuel economy, the policy that governs power distribution among HEV's energy sources is a determining factor. The very strategy also impacts other metrics such as drivability (Miro-Padovani et al., 2016), safety (Yu et al., 2015), component lifetime (Hannan et al., 2014), etc. HEV powertrain components are highly coupled, and it is usually impossible to affect an index without influencing others. While fuel economy and emissions are the mostly emphasized indices in designing energy man- 
agement strategies (EMS), a successful design has to consider other important metrics too. The holistic optimization of the powertrain is, as a result, an intricate problem to define and solve. Additionally, practical EMS design has to be implementable in real-time. This has encouraged significant amount of research in this area.

Despite the remarkable advancements in EMS design, there is still no comprehensive, silver bullet solution to this problem, and there are many questions left unanswered. This thesis is an endeavor to address a few of these questions for series hybrid electric vehicles (SHEV). The main application considered is a heavy duty piece of construction equipment, namely the Volvo's LX01 wheel loader. In what follows, the motivation behind the work and the key objectives are presented.

\subsection{Motivation}

Batteries comprise a large portion of HEV costs considering purchase price, maintenance, and replacement charges. Therefore, it is important to develop battery-friendly strategies that prolong battery lifetime so that the associated costs are reduced. This is particularly critical for vehicles with large batteries in which the battery systems are sized to be the main sources of propulsion. Such is usually the case for series HEVs. In fact, a SHEV can be viewed as an electric vehicle whose range and maximum power limits are extended by a generator set. Regarding the wheel loader, incorporation of battery life optimization into the energy management problem is crucial because (1) the battery is expensive considering its size, and (2) drive cycles can be aggressive, and if battery degradation is not reckoned with during EMS design, the strategy can considerably wear down the battery. As power requests are large in this application, remarkable improvements in battery lifetime can be expected, which in turn reduces total vehicle cost. This problem is addressed in the first paper within a non-predictive framework, namely Pontryagin's minimum principle.

Similar objectives can be defined for predictive frameworks such as model predictive control (MPC). While a similar outcome as before might be expected, there are fundamental differences between the employed frameworks. Performance of MPC depends, to a large extent, on control design parameters such as objective function's weights and prediction/control horizon. In particular, it is necessary for the prediction horizon to be long enough to guarantee acceptable results. For the energy management problem, the implication is that the prediction horizon should be adequately long with respect to the length of the drive cycle under study. This poses a challenge when the drive cycle is considerably long. For the wheel loader, a typical mission usually involves consecutive execution of one or more tasks, and the daily operation can be perceived as a single, prolonged drive cycle. Consequently, the prediction horizon might need to be unrealistically long to achieve desirable performance, defined over 
one day of work. This is, however, not a practical choice due to prediction inaccuracies and large computational burden. Formulation of the energy management problem, namely the compromise between fuel economy and battery life optimization, and the effects of different MPC design parameters are investigated in the second paper.

Energy management strategies are abundant in the literature. Rule-based methods are usually simple and easy to implement, but on the flip side, they exhibit unpredictable performance in dealing with real-world drive cycles. For better fuel economy, causal optimization-based approaches are proposed. A challenge pertained to these methods is computational complexity for real-time realization. One such method is the equivalent consumption minimization strategy (ECMS) which instantaneously minimizes a cost function (Paganelli et al., 2002). Even though ECMS is much less computationally demanding than a lot of the existing methods, its real-time implementation is still challenging (F. Zhang, L. Wang, et al., 2020). This observation sparked the motivation to analytically derive the ECMS solution by directly taking optimization constraints into account so as to alleviate the aforementioned problem by reducing computation time. This is performed in the third paper. Motivated by the findings of this work, the proposed analytical solution is used as a stepping stone to develop two real-time capable predictive strategies. The idea is to develop methods that are as computationally efficient as rule-based strategies, while maintaining performance of optimization-based approaches. It is of interest to investigate situations where no information on driving mission is known a priori, as well as cases where the vehicle is expected to follow, closely but not exactly, a set of predefined drive cycles, such as the wheel loader application.

While the hybrid wheel loader is the main application studied throughout the thesis, the proposed strategies are developed for a general SHEV powertrain. 



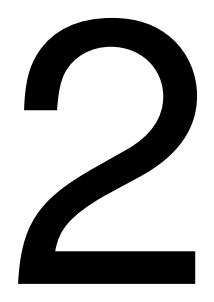

\title{
BACKGROUND
}

\author{
Problems that remain persistently \\ insoluble should always be \\ suspected as questions asked in the \\ wrong way.
}

Alan Watts

HEVs can be categorized based on the degree of hybridization into micro hybrids (start/stop feature), mild hybrids (start-stop plus braking recuperation), full hybrids (mild hybrid capability plus full-electric launch) and plug-in hybrids (full hybrid capabilities plus charging possibility via grid energy) (Guzzella et al., 2007). In another classification, depending on the powertrain configuration, HEVs can be categorized into series hybrids, parallel hybrids, and power split hybrids (Guzzella et al., 2007). In series hybrid, the engine drives a generator to produce electric power. The generated power is added to battery power, and the summation is fed to a motor that propels the vehicle. Thus, a series hybrid configuration can be seen as an electric vehicle equipped with a range-extender, characterized by serial connection of an engine and a generator. The main advantage is that the engine is mechanically decoupled from the wheels. This offers freedom in regulating engine speed to achieve high efficiency. Additionally, packaging and design is easier due to electrical connection of energy sources. The disadvantage is that three energy conversions are needed for the fuel power to reach the wheels, which decreases the 


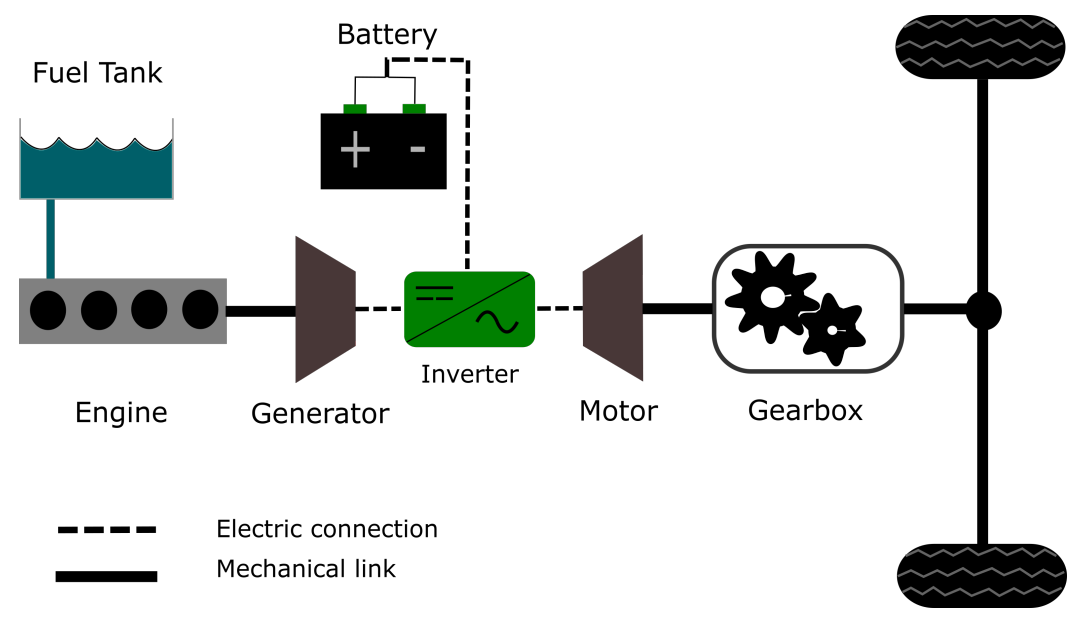

Figure 2.1: Series hybrid powertrain

powertrain efficiency and increases fuel consumption especially in highway driving. Fuel economy is usually better in parallel hybrids as only one energy conversion is needed for fuel power, unlike series hybrids. Also, the battery propels the vehicle in low speed scenarios to avoid low efficiency regions of engine operation. Nonetheless, there is less freedom in controlling engine operating points due to mechanical coupling to the wheels. As a result, this configuration tends to perform worse in stop-and-go traffic where the engine is inefficient. The torque split configuration combines the attractive features of series and parallel configurations and introduces an additional degree of freedom in that the engine and electric machines are decoupled, thanks to the power split device. The configuration considered in this thesis is the series hybrid, which is illustrated in Figure 2.1.

Successful performance of HEVs depends not only on the optimal design and sizing of the powertrain components, but also on the development of efficacious control systems that govern them (F. Zhang, L. Wang, et al., 2020). A key element of the latter is the energy management system that splits power request between the onboard energy sources. In what follows, a concise background on the most popular strategies will be given, and the methodologies that relate to the thesis will be briefly explained.

\subsection{Energy management strategies}

Depending on a given criterion, different classifications can be proposed for the existing energy management strategies. With respect to the computation scheme, they can be categorized into offline and online methods. Alternatively, according to 
the mechanism that determines the control action, EMSs can be put into groups of rule-based (or heuristic) and optimization-based methods. The methodologies can also be classified based on exploitation of future driving condition predictions to non-predictive and predictive strategies. It should be noted that methods of different categories are not mutually exclusive. In fact, improvements in performance can be achieved through combining different energy management methods. In the following, a brief explanation on each group is given.

\section{Offline methods}

Some of the methods in this category globally optimize a cost function that can take into account different design objectives. Examples are dynamic programming (DP), genetic algorithm (GA), and game theory (Dextreit et al., 2008; Panday et al., 2016; Serrao et al., 2011). These methods are generally computationally exhaustive and require complete knowledge of future driving conditions, and hence they cannot be implemented in real-time. They are usually regarded as benchmarks to evaluate performance of other methods. In addition, they can be used to develop rule-based methods by extracting heuristic policies (Peng et al., 2017). Suboptimal results can be found by predicting future drive cycle in order to make them suitable for real-time implementation. For example, stochastic dynamic programming (SDP) predicts future speed/power demand by stochastic methods such as Markov chain and performs the backward induction scheme of DP on the prediction (Daniel F Opila et al., 2013). Still, the high computational complexity of the method discourages its usage in real-time (F. Zhang, L. Wang, et al., 2020).

Pontryagin's minimum principle (PMP) is another well-known method adopted from optimal control theory to address the energy management problem (Yuan et al., 2013). It is essentially a two-point boundary value problem that yields locally optimal solutions. Even though this method transforms the optimization over the whole drive cycle to an instantaneous minimization of a function, determination of the costate requires future drive cycle knowledge, which makes it non-causal. The initial value of costate is usually computed by iteration-based algorithms such as shooting methods (Cristiani et al., 2010).

Rule-based methods might be considered as a subcategory of the offline group of EMSs as they are designed in offline settings (F. Zhang, L. Wang, et al., 2020). These methods are popular since they are easily implementable. On the flip side, their performance can suffer greatly if the real-world drive cycles are quite different from the ones used to derive the controller rules. 


\section{Online methods}

Online EMSs do not require complete knowledge of drive cycle. They obtain suboptimal results, but they offer the possibility of real-time implementation. They can be categorized into instantaneous optimization-based and predictive strategies.

\section{Instantaneous optimization-based EMS}

Instantaneous optimization-based methods do not exploit future drive cycle prediction. They determine the control policy according to instantaneous values of the powertrain operating points. They include equivalent consumption minimization strategy (ECMS), adaptive ECMS, and robust control.

In ECMS, the idea is to minimize, at each sample, the total fuel power given as the summation of the fuel power consumed by the internal combustion engine (ICE) and the equivalent fuel power of the electric storage system (ESS), which is the battery in our case. The total fuel consumption rate is computed as

$$
P_{f, t o t}=P_{f}+s P_{e c h}
$$

where $P_{f}$ is the ICE fuel power, $P_{e c h}$ is the battery electrochemical power, $s$ is the equivalence factor, and $P_{f, t o t}$ is the total fuel power. The intuition underpinning this idea can be explained by the charge sustainability constraint, which requires battery charge to be sustained at a reference value by the end of drive cycle. A battery that is discharged below the reference value has to be compensated for in future by excessive fuel consumption. Conversely, charging the battery above a reference value can save fuel in future. Thus, an equivalence can be established between the power coming from fuel and the stored power of the battery. Variable $s$ governs this equivalence, and it is determined such that charge sustenance is fulfilled. As $s$ is sensitive to the drive cycle under consideration, its exact value can be determined only if the drive cycle is known beforehand, which is not the case in practice. Thus, the main challenge of ECMS lies in the regulation of equivalence factor (Musardo et al., 2005). Adaptive ECMS methods, also known as A-ECMS, are hence proposed to make ECMS more adaptable to real world drive cycles. These methods are computationally more efficient than the non-causal methods, but their implementation can pose challenges. As a result, they might be implemented as look-up tables.

The most popular A-ECMS methods use proportional (P) or proportional-integral (PI) controllers to control $s$ based on instantaneous value of the state of charge (SOC) as

$$
s(t)=s_{0}+k_{P}\left(\operatorname{SOC}_{r e f}(t)-\operatorname{SOC}(t)\right)+k_{I} \int_{0}^{t}\left(\operatorname{SOC}_{r e f}(\tau)-\operatorname{SOC}(\tau)\right) \mathrm{d} \tau,
$$


where $k_{P}$ and $k_{I}$ are control parameters, $s_{0}$ is an initial value, and $\mathrm{SOC}_{r e f}$ is the reference value for the state of charge ( $\mathrm{Gu}$ et al., 2006). It should be noted that SOC $_{r e f}$ is not necessarily constant (Tianheng et al., 2015). The developed algorithms perform best when real world drive cycles mimic the ones used to tune the control parameters, otherwise the performance might diminish. To tackle this issue, intelligent transportation systems can be employed to improve A-ECMS performance. For instance, information from vehicle-to-vehicle (V2V) and vehicle-to-infrastructure (V2I) communications can be exploited to enhance accuracy of velocity forecast, which is in turn used for better adjustment of controller parameters (F. Zhang, Xi, et al., 2017).

\section{Predictive EMS}

Unlike the instantaneous optimization-based EMSs, methods in this category optimize the power split over a future horizon based on velocity and/or power predictions. A powerful tool is model predictive control. The idea is to optimize an objective function, defined over a certain horizon, at each time step. The first element of the optimal control signal is fed into the system, and the problem is repeated in a receding horizon scheme. MPC can thus be seen as a compromise between DP and ECMS, as it uses partial predictions of drive cycle. Some advantages of MPC are as follows (Huang et al., 2017):

- System constraints can be directly taken into account for optimization.

- Computational complexity does not increase for multivariable systems compared to single-input single-output systems since MPC algorithms usually use state-space representation.

- There is freedom in designing objective function to include desirable objectives.

- MPC uses instantaneous feedback and has nominal robustness as a result.

- Future information can be utilized to enhance results.

Consider the system described by

$$
\begin{aligned}
\mathbf{x}_{k+1} & =f\left(\mathbf{x}_{k}, \mathbf{u}_{k}\right), \\
\mathbf{y}_{k} & =h\left(\mathbf{x}_{k}, \mathbf{u}_{k}\right),
\end{aligned}
$$

where $\mathbf{x}_{k}, \mathbf{y}_{k}$, and $\mathbf{u}_{k}$ are respectively the state vector, the output vector, and the input vector at sample $k$, and $f$ and $h$ are nonlinear functions. A generic objective function for MPC can be described by (Rawlings et al., 2017)

$$
V\left(\mathbf{x}_{0}\right)=\sum_{k=0}^{N-1} l\left(\mathbf{x}_{k}, \mathbf{u}_{k}, k\right)+V_{N}\left(\mathbf{x}_{N}\right),
$$


where $\mathbf{x}_{0}$ is the system state at current time, $N$ is the control horizon, $V$ is the objective function to be minimized, $l$ is a nonlinear function, and $V_{N}$ is the terminal penalty designed to maintain stability. The goal is to find the admissible input sequence $\left\{\mathbf{u}_{0}, \mathbf{u}_{1}, \ldots, \mathbf{u}_{N-1}\right\}$ that minimizes (2.4), subject to (2.3). In energy management problem, the output vector consists of fuel mass flow rate which is a nonlinear function of inputs and system states. In this case, the cost function consists of terms that correspond to fuel consumption and SOC (and other important variables) in order to minimize fuel consumption and enforce charge sustenance. A common cost function is

$$
J=\sum_{k=0}^{N-1}\left(\dot{m}_{f, k}^{2}+\alpha_{k}\left(\mathrm{SOC}_{k}-\mathrm{SOC}_{r e f}\right)^{2}\right)+\alpha_{N}\left(\mathrm{SOC}_{N}-\mathrm{SOC}_{r e f}\right)^{2},
$$

where $\dot{m}_{f, k}$ and $\mathrm{SOC}_{k}$ are respectively the fuel mass flow rate and battery state of charge at sample $k$ in the horizon, and $\alpha_{i}, i=0,1, \ldots, N$, are weights. For the energy management problem, the time dependency of stage cost $l(\cdot)$ in (2.4), corresponds to time dependency of drive cycle prediction.

The energy management problem is inherently a nonlinear program. System dynamics can be linearized so that the optimization problem is transformed into a linear timevarying MPC. Including other objectives such as gear shift strategy and engine on/off introduces discrete variables and turns the formulation into a hybrid MPC which is computationally expensive to solve.

Success of predictive strategies depends, to a large extent, on the quality of future drive cycle predictions (Zhou et al., 2019). There are plenty of studies on effective prediction methods, which will be discussed later in this chapter. Yet, prediction inaccuracies are inevitable in practice. To deal with uncertainties that originate from predictions and also the ones rooting from system modeling imperfections, robust MPC methods such as tube-based MPC have been employed to design promising EMSs (Joševski et al., 2016; Sotoudeh et al., 2020). Another technique is stochastic MPC (SMPC) which hires stochastic framework to predict future driving cycle (Zeng et al., 2015). For example, power demand can be modeled as a Markov chain, which is trained offline using drive cycle data sets. Adaptability of SMPC to real-world drive cycles can be improved through updating the Markov chain in order to capture the varying driver behavior and traffic conditions (Di Cairano et al., 2013). Due to its computational complexity, application of SMPC in practice is still limited.

Similar to model predictive control, ECMS and PMP can also be applied on the predicted future power/torque request in a receding horizon fashion (J. Han et al., 2017; Xie et al., 2019). 


\section{Rule-based methods}

The rule-based techniques are developed through expert knowledge, intuition and extracting results from global optimization methods. The control signals are generated according to pre-determined rules.

Rule-based methods can be further classified as deterministic rule-based and fuzzy logic-based methods. Popular examples of deterministic rule-based methods are on-off and power follower strategies (Gao et al., 2009; Jalil et al., 1997). These techniques are attractive and popular due to simplicity of implementation. However, they might exhibit poor performance in dealing with real-world driving conditions that are different from the drive cycles used to extract rules. One remedy is to incorporate predictive schemes and utilize information from drive cycle prediction and recognition algorithms. The other group of rule-based methods are based on fuzzy logic rules (Mohebbi et al., 2005; Schouten et al., 2002; Tie et al., 2013). They promise better adaptability to new drive cycles and less sensitivity to uncertainties compared to deterministic methods and can be further improved through employment of artificial intelligence techniques (Chen et al., 2008). The main challenge of these methods is in choosing appropriate membership functions.

\section{Optimization-based methods}

Unlike the rule-based techniques, optimization-based methods compute control action through mathematical optimization of performance indices. They can be categorized into offline methods, such as DP and PMP, and online methods, such as MPC, ECMS, A-ECMS, etc. Also, these methods can either globally or locally optimize the performance objectives. Examples of global methods are DP and GA, while PMP and MPC are examples of local optimizers.

\section{Learning algorithms in EMS}

Unpredictable driving conditions such as traffic congestion and driving habits increase uncertainty of drive cycle prediction. Learning algorithms can be used to mitigate this problem. An example is given in Di Cairano et al. (2013), where the Markov model-based drive cycle predictor is updated to adapt to the changing driver behavior. Additionally, machine learning and reinforcement learning techniques can be employed to design model-free controllers to offer better adaptability compared to conventional EMSs (X. Han et al., 2019; Lian et al., 2020; T. Liu, Hu, et al., 2017). Real-time capable strategies can be designed via reinforcement learning so that the optimal decisions are learned from historical drive cycle data (Qi, G. Wu, et al., 2016). 
Clearly, this requires large library of data for training. The learning process can be made adaptive as well so that changes in driving conditions are taken into account (Qi, Luo, et al., 2017). Needless to say, the achieved fuel consumption figures are suboptimal.

\section{Drive cycle prediction methods}

As it was mentioned previously, the accuracy of future driving condition predictions is a determining factor in guaranteeing success of predictive strategies. It is impossible to perfectly know the future conditions, but one can regard the prescient scenario, where future driving conditions are exactly known, as a benchmark against which performances of causal prediction methods are assessed. In the sequel, some of the most popular prediction techniques, including the stochastic method used in paper IV, are touched upon.

\section{Frozen-time prediction}

The simplest prediction scheme is to assume constant power demand over the prediction horizon (Banvait et al., 2013). This assumption can make control design simpler, however, its prediction accuracy is usually poor specially for scenarios with frequent power request fluctuations.

\section{Exponentially-varying prediction}

In this method, either torque, power demand or vehicle velocity is predicted according to an exponential formula (C. Sun, Hu, et al., 2014a) over the optimization horizon. For instance, torque can be assumed to behave according to

$$
\hat{T}(k)=T(0) \exp (-\tau k),
$$

where $\hat{T}(k)$ is the torque request at $k^{\text {th }}$ sample in the horizon, $T(0)$ is the torque request at current time, and $\tau$ is a decay rate. Vehicle velocity can be found, if required, through its relationship with demanded torque. Alternatively, the velocity can be predicted according to a formula. For example, C. Sun, Hu, et al. (2014b) uses

$$
\hat{v}(k)=v(0)(1+\varepsilon)^{k}
$$

for velocity forecast. In (2.7), $\hat{v}(k)$ is the velocity at $k$ th sample in the horizon, $v(0)$ is the velocity at current time, and $\varepsilon$ is an exponential coefficient. 
Prediction accuracy decreases when torque request (or speed trajectory) does not follow the exponential trend. In particular, (2.6) has the worst performance when torque is increasing throughout the horizon.

\section{Stochastic modeling}

Future power demand (or velocity) can be modeled as a stochastic process. Markov chains are quite popular for this purpose (T. Liu, B. Wang, et al., 2018). In this case, it is assumed that power demand satisfies the Markov property, i.e., at any given time, power demand only depends on its value at the previous instant. Consider a discrete-time stochastic process $\left\{X_{k}, k \in \mathbb{Z}_{\geqslant 0}\right\}$, with the finite state space $W=$ $\left\{w_{1}, w_{2}, \ldots, w_{m}\right\}$. In mathematical terms, the Markov property states that for any $k \in \mathbb{N}$ and $l \in \mathbb{N}_{\leqslant k}$,

$$
\operatorname{Pr}\left(X_{k}=x_{k} \mid X_{k-1}=x_{k-1}, \ldots, X_{k-l}=x_{k-l}\right)=\operatorname{Pr}\left(X_{k}=x_{k} \mid X_{k-1}=x_{k-1}\right),
$$

where $x_{i} \in W$ for $i=k-l, k-l+1, \ldots, k$.

To model power demand as a Markov process, the admissible range of power requests is discretized and divided into several intervals according to a certain step (Di Cairano et al., 2013). Each interval can be represented by the average value of its bounds. Considering $m$ intervals, the set of possible states would then be $\mathscr{P}=\left\{\mathscr{P}_{1}, \mathscr{P}_{2}, \ldots, \mathscr{P}_{m}\right\}$, where $\mathscr{P}_{i}$ is the average value of power in $i^{\text {th }}$ interval. Once a set of training data is available, transition probability matrix is constructed as $T=\left[t_{i j}\right]_{m \times m}$, in which

$$
\begin{aligned}
t_{i j} & =\operatorname{Pr}\left(P_{\text {req }}(k+1)=\mathscr{P}_{j} \mid P_{\text {req }}(k)=\mathscr{P}_{i}\right), \\
& =\frac{n_{i j}}{n_{i}},
\end{aligned}
$$

where $n_{i j}$ is the number of transitions from $\mathscr{P}_{i}$ to $\mathscr{P}_{j}$ and $n_{i}$ is the number of times $\mathscr{P}_{i}$ has occurred. Clearly, $\sum_{j=1}^{m} t_{i j}=1$.

The size of $\mathscr{P}$ determines the trade-off between prediction precision and computational load. The transition probability matrix is found by maximum likelihood estimation, using drive cycle data and is then used to predict future power demand up to a certain horizon (L. Liu et al., 2012). To capture variations due to driver behavior and traffic conditions, the Markov model can be updated via a learning algorithm (Di Cairano et al., 2013). The quality of predictions depends on the resemblance of real-world drive cycles to the ones used to train the Markov chain models. 


\section{Artificial intelligence-based methods}

Artificial intelligence (AI) methods are extensively used for drive cycle prediction and have the ability to outperform parametric and stochastic methods (C. Sun, Hu, et al., 2014b). Examples are neural networks, Fuzzy logic pattern recognition and support vector machine (Moser et al., 2015; Y. Zhang, 2010). The idea is to extract features from a large database of drive cycle segments, and then employ an AI method to recognize the driving pattern ahead (Jeon et al., 2002; Langari et al., 2005; Murphey et al., 2008; J. Wu et al., 2012). Such techniques are appealing for fixed-route vehicles and also vehicles that follow predefined drive cycles such as construction machinery. Prediction fidelity depends on the drive cycles used to train pattern recognition algorithms.

\section{Telematics}

Better prediction accuracy can be achieved by utilizing extra information from intelligent transportation systems such as global position system (GPS), geographic information system (GIS), real-time traffic signal data, vehicle-to-vehicle (V2V) and vehicle-to-infrastructure (V2I) communication, and traffic flow modeling (Gong et al., 2008; Keulen et al., 2010; Zhou et al., 2019; Zulkefli et al., 2014). The information can be used to determine vehicle's position, predict road traffic, and gather information on road terrain to improve velocity and power prediction (C. Zhang et al., 2009). Furthermore, the information can be exploited to generate speed profiles and reference SOC trajectories to be used in EMSs (He et al., 2012; C. Sun, F. Sun, et al., 2015). Velocity can be predicted using GPS and a stored library of velocity trajectories, each corresponding to a specific vehicle position (Bartholomaeus et al., 2008). For better adaptability to driver behavior changes, drive cycle library can be kept updated in real-time. Traffic flow modeling methods have also been used to produce better predictions (Gipps, 1981). Telematics and AI methods can be merged in order to enhance accuracy.

\section{Integration of other objectives into EMS}

Improvement in fuel economy and reduction in greenhouse gas emissions are usually the main objectives considered in EMS design. EMS policies affect individual performance of powertrain components. As a result, other metrics that are influenced by powertrain operating points might be negatively affected and should therefore be taken into consideration during the EMS design process (Daniel F. Opila et al., 2012). An important factor is drivability. While there is no single unanimouslyaccepted definition for it, engine on-off switching and gear shift are the aspects 
usually considered to incorporate the drivability notion (Miro-Padovani et al., 2016). Inclusion of these variables into EMS makes the problem more complex. Some of the popular methods are convex optimization (Elbert et al., 2014), optimal control (Ngo et al., 2012), and artificial intelligence (Li et al., 2019).

From the economic perspective, fuel consumption is not the only deciding factor for total cost. Powertrain components maintenance and replacement fees constitute a large portion of HEV expenses. Therefore, component durability should also be incorporated into EMS. In particular, it is important to mitigate battery wear to reduce costs (Ebbesen et al., 2012; Tang et al., 2015). Degradation of battery occurs when it is at rest and also through cycling, which manifests itself as capacity fade, power fade, etc. Conventionally, battery end of life (EOL) is marked as the time capacity fades down to 80 percent of its initial value. Hence, to include battery life optimization into the energy management problem and predict the EOL, a common approach is to use a capacity fade model. Empirical and semi-empirical models are appropriate candidates for this purpose due to simplicity and reasonable computational effort needed for simulation, compared to their electrochemical counterparts (Jin et al., 2018). One such control-oriented model is used in this thesis for integration of battery wear into the energy management problem (Suri et al., 2016).

\subsection{Summary and contributions}

In what follows, the included papers are summarized, and the contributions are outlined. The first two papers deal with incorporation of battery aging mitigation into the energy management problem. The aging phenomena are complicated to mathematically describe, and hence a simple control-oriented model is used for this purpose. The last two papers are motivated by the need to develop real-time energy management strategies. The key idea of these papers is to exploit analytical solution formulas to reduce computation time. The hybrid wheel loader is the assessment application in all papers, but the strategies are developed for series hybrid electric powertrains, regardless of vehicle operation.

\section{Paper I}

Shafikhani, Iman and Jan Åslund (2021). "Energy management of hybrid electric vehicles with battery aging considerations: Wheel loader case study". In: Control Engineering Practice 110, p. 104759.

Integration of battery degradation reduction into the energy management problem of series hybrid electric vehicles is investigated in this work. The genset dynamics are neglected as they are fast compared to supervisory controller's sampling time. This 
assumption is made through all papers. Therefore, the genset is modeled as a static map, constructed from high-fidelity, experimentally validated engine and generator models.

Battery degradation is included in the EMS design using a capacity fade model. The original batch model was introduced in Suri et al. (2016), which uses standard constant C-rate aging cycles. As the battery operating points vary a lot in a realworld application, the model is differentiated with respect to time in order to make it accessible for simulation. This hypothesis is based on the investigation done in Jin et al. (2018), which suggests that differentiated batch models might offer proper choices for modeling capacity fade for varying operating point scenarios. A two-step algorithm is proposed to identify the differential capacity fade model parameters from constant C-rate battery aging experiments. The load cycles do not need to be symmetric, i.e., the charge and discharge C-rates can be different. Experimental data is then used to find the model parameters for the wheel loader application.

The energy management problem is formulated within the optimal control framework and is solved using Pontryagin's minimum principle. To make the performance objectives numerically comparable, the expressions for fuel consumption and capacity fade are normalized with respect to reference values. The terms are further weighted with respect to each other via parameter $a \in[0,1]$ as the weight to be designed. These ideas are adopted from Tang et al. (2015).

Simulations are performed for four drive cycles, namely short loading cycle (SLC), load and carry (LC), load and carry uphill (LCU), and fork palletizing (FP). In simulations done for each standard drive cycle, it is observed that when battery lifetime is included in the optimization cost function, corresponding to $a \neq 1$, the PMP solution leads to "charge less, assist less" policy for the battery, compared to the case where only fuel consumption is minimized. Additionally, brake power regeneration is almost perfect for $a \neq 1$.

Simulations, spanning one full year of operation, are carried out in order to predict and compare long-term performances resulting from different choices of parameter $a$. The results suggest that battery lifetime can be improved by $13 \%$ to $55 \%$ depending on the drive cycle, and the cost of achieving such result is a negligible increase in fuel consumption. It is also revealed that the battery lifetime improvements are less pronounced for drive cycles in which power requests are larger.

\section{Paper II}

Shafikhani, Iman, Christofer Sundström, Jan Åslund, and Erik Frisk (2021).“MPCbased energy management system design for a series HEV with battery life optimizatio”. In: European Control Conference. 
Similar objectives as in paper $\mathbf{I}$ are pursued in this work. The idea is to include battery lifetime optimization into the energy management problem in a predictive framework. One might expect similar findings as in paper $\mathbf{I}$, but there is a fundamental difference between these scenarios: the performance of predictive strategies largely depends on the control parameters such as the optimization horizon. It is well-known that the optimization horizon should be large enough to yield acceptable performance. For the hybrid wheel loader, one day of operation usually includes numerous repetitions of one or more cycles. The whole operation can be thus regarded as one long-duration drive cycle. For the predictive EMS to have decent performance, defined over one day of operation, the optimization horizon should be large enough compared to the length of the drive cycle that lasts eight hours. However, this may not be practical as the optimization horizon might get unreasonably large. This consideration motivated the work done in paper II.

Model predictive control, as a popular framework for predictive optimization, is employed to define and solve the energy management problem. The same powertrain component models as before are used in this paper, except for the genset for which a logarithmic model is introduced in order to avoid solving a mixed integer program due to engine on-off status. The main reason for doing so was to reduce computation time and keep the possibility of real-time implementation. Similar ideas of normalization of performance expressions with respect to reference values and introduction of the weighting parameter as in paper $\mathbf{I}$ is used to construct the performance index. To make the optimization problem simple, a quadratic cost function is introduced. Furthermore, as large battery currents - and thus large battery power values - accelerate battery aging, the square of battery terminal power is penalized in the cost function to minimize capacity degradation, instead of the capacity fade rate that was used in paper $\mathbf{I}$.

The parameter that determines the relative weight between fuel consumption and capacity degradation terms is denoted by $\sigma \in[0,1]$, where $\sigma=1$ corresponds to the scenario where the optimization only includes the fuel consumption term. Simulations are performed for different values of $\sigma$, and two methods, namely nonlinear MPC and linear time-varying MPC, are used to solve the problem with three optimization horizons of 10, 40 and 100 samples.

At first it is assumed that the drive cycles are perfectly known. It is observed that battery lifetime is improved as $\sigma$ is reduced, which was expected. Additionally, the best fuel economy is not achieved by $\sigma=1$. This means that it is possible to adjust $\sigma$ such that fuel consumption and battery lifetime are improved at the same time. For the values of $\sigma$ that yield the best fuel economy for each of the six scenarios, denoted by $\sigma^{*}$, the battery lifetime improvements are between 40 and 58 percent, compared to the benchmark for which the prediction horizon is 100 samples, $\sigma=1$, and nonlinear MPC is the employed optimization method. In all cases, the fuel economy figures 
corresponding to $\sigma^{*}$ is better than the aforementioned benchmark. The differences, however, are insignificant.

Similar simulations are performed for scenarios where large drive cycle uncertainties exist. The methods exhibit robustness against the randomly introduced uncertainties, and the $\sigma^{*}$ values corresponding to the prescient scenarios are close to the ones that yield the best fuel economy for the uncertain cases. Battery lifetime improvements corresponding to prediction horizon of 10 samples are slightly more than 30 percent with respect to the benchmark.

Proposition of a real-time implementable method was not the goal of paper II, yet the findings suggest that it is possible to reduce computation time by reducing the prediction horizon and employing a time-efficient algorithm such as linear time-varying MPC, while gaining performance in terms of fuel economy and battery lifetime.

\section{Paper III}

\section{Shafikhani, Iman and Jan Åslund (2021).“Analytical Solution to Equivalent Consumption Minimization Strategy for Series Hybrid Electric Vehicle”. In: IEEE Transactions on Vehicular Technology 70.3, pp. 2124-2137.}

Analytical solution to equivalent consumption minimization strategy for SHEVs was investigated in Luján et al. (2018), where admissibility of the control variable was not thoroughly taken into consideration. Paper III is dedicated to comprehensive exploration of the same problem. This is an important step in the development of computationally feasible EMSs carried out in this thesis. Positive power demands are partitioned into four disjoint sets, and the analytical solution is computed for each set separately. It is found that the shape of the analytical solution, in power demandequivalence factor (EF) plane, depends on an intrinsic property of the powertrain. The proposed solution is calculated as a function of the powertrain model parameters; thus, the solution is automatically adapted when the model parameters vary. Furthermore, the proposed policy is valid regardless of the adaptation mechanism that controls the EF. Validity of the analytical policy is evaluated for the wheel loader for two drive cycles. It is observed that the proposed analytical solution is about 45 times faster than numerical optimization.

From the analytical solution, the effective bounds of EF are computed. It is demonstrated that varying the EF outside of these bounds does not bring about change in the ECMS solution. The effective bounds are used to propose an adaptive ECMS for the wheel loader. For each drive cycles, only one value of the EF, corresponding to the standard drive cycle, is used in construction of the EF controller. The methodology regulates the EF, based on which the analytical policy computes the optimal (in the sense of ECMS) genset/battery power. Simulations are performed for 12 drive cycles that are constructed by random induction of uncertainty in the standard drive cycles. 
It is observed that the fuel consumption values achieved by the proposed methodology are very close to the values found by PMP. In addition, the methodology is charge sustaining for all drive cycles.

\section{Paper IV}

Shafikhani, Iman, Erik Frisk, Jan Åslund, and Christofer Sundström (2021). "Real-time Predictive Energy Management Strategies for Series Hybrid Electric Vehicles". Manuscript.

In this paper, two predictive ECMS algorithms are developed for SHEVs. Fuel economy and computation time efficiency are the key elements considered in EMS development. Results of paper III are the basis for the algorithms developed in this work. Two different scenarios are explored. In the first scenario, applications that are expected to follow certain drive cycles are dealt with. For such vehicles, prior information about driving mission is available, and real-world drive cycle data is usually accessible. The electric power demand is modeled as a Markov chain, which is trained using the drive cycle data. At any sampling time, the idea is to find the EF such that ECMS is charge sustaining for the first positive portion of the predicted power demand. If charge sustenance cannot be achieved, EF is regulated such that the terminal state of charge is as close to a reference value as possible. Determination of the EF is based on linear interpolation between the EF bounds and two other special values introduced in paper III. Once the EF is computed, the analytical solution proposed in paper III is used to determine the control input corresponding to the current time. In the second scenario, it is assumed that no prior information is known about drive cycles. This is a common situation encountered in urban driving. The future power demand is modeled as a linear trend according to the power demand values at current time and the previous sample. The optimal EF is calculated by solving the charge sustenance equation in continuous-time analytically.

The developed methods are simulated for the hybrid wheel loader and a passenger car. Simulation results for the wheel loader for five drive cycles demonstrate that both algorithms achieve fuel consumption figures close to those found through dynamic programming. Furthermore, the computation times of both methods are small enough to make them feasible for real-time implementation. For the passenger car, the proposed algorithm corresponding to the second scenario is simulated for five popular drive cycles, and the results are compared to dynamic programming, thermostat rule-based and power follower rule-based strategies (Gao et al., 2009). It is observed that the presented algorithm obtains satisfactory fuel economy compared to DP, and unlike the rule-based strategies, it is charge sustaining for all drive cycles. 


\subsection{Reflection and future research outlook}

The first two works hint at the potential in significant prolongation of battery lifetime, where the compromise on fuel consumption is negligible to none, depending on the optimization framework. While this result might encourage implementation of the developed methodologies, several key questions are left unanswered.

The system models used in the control design process are simplified. For a more accurate analysis, therefore, it seems crucial to perform the same investigations, using more detailed models of the powertrain components. This also includes the aging model used to represent capacity degradation. In particular, power fade is a consequence of battery wear, which was not embedded in the employed aging model and optimization constraints. Furthermore, robustness of the methods with respect to uncertainties arising from model inaccuracies and unmodeled dynamics have not been studied in the papers.

In paper I, a lookup table for computation of the equivalence factor was developed as a function of initial battery state and initial maximum capacity. As real-world drive cycles are different from those used to develop such lookup table, it is essential to investigate performance of the method when drive cycle uncertainties are present. In the same work, the appropriate value of parameter $a$ was decided upon considering the fuel and battery costs, where the latter only included the initial purchase price of the battery. Other expenses such as maintenance and replacement fees should also be incorporated in the analysis.

Numerous rule-based energy management strategies exist that emphasize on fuel consumption minimization, but the literature seems to be lacking in rule-based methods that take battery degradation into consideration. In this regard, the results of paper I and II might be used to extract heuristic strategies.

Papers III and IV demonstrate the advantage of using analytical solution in development of efficient EMSs. Since the system constraints are explicitly taken into account in derivation of the policy, the computation time is significantly reduced for online implementation. The same procedures can be done for multi-objective energy management, using more accurate models of the powertrain.

The methods presented in papers III and IV can be further improved by including other objectives. For instance, engine on-off optimization is an essential feature that should be considered in EMS design. Thus, reduction in number of engine on-off switching should be incorporated in the proposed methods. To do so, one idea is to equip the established algorithms with heuristic rules. Moreover, performance of predictive EMSs is sensitive to the quality of drive cycle prediction. The data acquired from intelligent transportation systems can be utilized to enhance prediction accuracy in order to improve performance of the EMSs developed in paper IV. 
For the wheel loader under study, the analysis carried out in all papers can be further extended by integrating the productivity index, defined by the amount of material being loaded, transported, etc., into the energy management problem as a new optimization variable. This means that the electric power demand sequence that represents the drive cycle can be regarded as an additional control variable. Nevertheless, it is not trivial how to mathematically describe the productivity index and how to convert it to an equivalent cost so that it can be compared to fuel and battery costs. 



\section{BIBLIOGRAPHY}

Banvait, Harpreetsingh, Jianghai Hu, and Yaobin Chen (2013). "Energy management control of plug-in hybrid electric vehicle using hybrid dynamical systems". In: IEEE Transactions on Intelligent Transportation Systems.

Bartholomaeus, Ralf, Matthias Klingner, and Martin Lehnert (2008). "Prediction of power demand for hybrid vehicles operating in fixed-route service”. In: IFAC Proceedings Volumes 41.2, pp. 5640-5645.

Chen, ZhiHang, M Abul Masrur, and Yi L Murphey (2008). "Intelligent vehicle power management using machine learning and fuzzy logic". In: 2008 IEEE International Conference on Fuzzy Systems (IEEE World Congress on Computational Intelligence). IEEE, pp. 2351-2358.

Cristiani, Emiliano and Pierre Martinon (2010). "Initialization of the shooting method via the Hamilton-Jacobi-Bellman approach”. In: Journal of Optimization Theory and Applications 146.2, pp. 321-346.

Dextreit, C, F Assadian, IV Kolmanovsky, J Mahtani, and K Burnham (2008). Hybrid electric vehicle energy management using game theory. Tech. rep. SAE Technical Paper.

Di Cairano, Stefano, Daniele Bernardini, Alberto Bemporad, and Ilya V Kolmanovsky (2013). "Stochastic MPC with learning for driver-predictive vehicle control and its application to HEV energy management". In: IEEE Transactions on Control Systems Technology 22.3, pp. 1018-1031.

Ebbesen, Soren, Philipp Elbert, and Lino Guzzella (2012). "Battery state-of-health perceptive energy management for hybrid electric vehicles". In: IEEE Transactions on Vehicular technology 61.7, pp. 2893-2900.

Elbert, Philipp, Tobias Nüesch, Andreas Ritter, Nikolce Murgovski, and Lino Guzzella (2014). "Engine on/off control for the energy management of a serial hybrid electric bus via convex optimization". In: IEEE Transactions on Vehicular Technology 63.8, pp. 3549-3559.

Gao, Jianping, Fengchun Sun, Hongwen He, Guoming G Zhu, and Elias G Strangas (2009). "A comparative study of supervisory control strategies for a series 
hybrid electric vehicle". In: 2009 Asia-Pacific Power and Energy Engineering Conference. IEEE, pp. 1-7.

Gipps, Peter G (1981). "A behavioural car-following model for computer simulation". In: Transportation Research Part B: Methodological 15.2, pp. 105-111.

Gong, Qiuming, Yaoyu Li, and Zhong-Ren Peng (2008). "Trip based optimal power management of plug-in hybrid electric vehicles using gas-kinetic traffic flow model". In: 2008 American control conference. IEEE, pp. 3225-3230.

$\mathrm{Gu}, \mathrm{Bo}$ and Giorgio Rizzoni (2006). "An adaptive algorithm for hybrid electric vehicle energy management based on driving pattern recognition". In: ASME International Mechanical Engineering Congress and Exposition. Vol. 47683, pp. 249-258.

Guzzella, Lino, Antonio Sciarretta, et al. (2007). Vehicle propulsion systems. Vol. 1. Springer.

Han, Jihun, Dongsuk Kum, and Youngjin Park (2017). "Synthesis of predictive equivalent consumption minimization strategy for hybrid electric vehicles based on closed-form solution of optimal equivalence factor". In: IEEE Transactions on Vehicular Technology 66.7, pp. 5604-5616.

Han, Xuefeng, Hongwen He, Jingda Wu, Jiankun Peng, and Yuecheng Li (2019). "Energy management based on reinforcement learning with double deep Qlearning for a hybrid electric tracked vehicle”. In: Applied Energy 254, p. 113708.

Hannan, MA, FA Azidin, and Azah Mohamed (2014). "Hybrid electric vehicles and their challenges: A review". In: Renewable and Sustainable Energy Reviews 29, pp. $135-150$.

He, Yiming, Jackeline Rios, Mashrur Chowdhury, Pierluigi Pisu, and Parth Bhavsar (2012). "Forward power-train energy management modeling for assessing benefits of integrating predictive traffic data into plug-in-hybrid electric vehicles". In: Transportation Research Part D: Transport and Environment 17.3, pp. 201-207.

Huang, Yanjun, Hong Wang, Amir Khajepour, Hongwen He, and Jie Ji (2017). "Model predictive control power management strategies for HEVs: A review". In: Journal of Power Sources 341, pp. 91-106.

Jalil, Nashat, Naim A Kheir, and Mutasim Salman (1997). "A rule-based energy management strategy for a series hybrid vehicle". In: Proceedings of the 1997 American Control Conference (Cat. No. 97CH36041). Vol. 1. IEEE, pp. 689-693.

Jeon, Soon-il, Sung-tae Jo, Yeong-il Park, and Jang-moo Lee (2002). "Multi-mode driving control of a parallel hybrid electric vehicle using driving pattern recognition". In: J. Dyn. Sys., Meas., Control 124.1, pp. 141-149.

Jin, Xing, Ashish Vora, Vaidehi Hoshing, Tridib Saha, Gregory Shaver, Oleg Wasynczuk, and Subbarao Varigonda (2018). "Applicability of available Liion battery degradation models for system and control algorithm design". In: Control Engineering Practice 71, pp. 1-9.

Joševski, Martina and Dirk Abel (2016). "Tube-based MPC for the energy management of hybrid electric vehicles with non-parametric driving profile prediction". In: 2016 American Control Conference (ACC). IEEE, pp. 623-630. 
Keulen, Thijs van, Bram de Jager, A Serrarens, and M Steinbuch (2010). "Optimal energy management in hybrid electric trucks using route information”. In: Oil \& Gas Science and Technology-Revue de l'Institut Français du Pétrole 65.1, pp. 103-113.

Langari, Reza and Jong-Seob Won (2005). "Intelligent energy management agent for a parallel hybrid vehicle-part I: system architecture and design of the driving situation identification process". In: IEEE transactions on vehicular technology 54.3, pp. 925-934.

Li, Guoqiang and Daniel Görges (2019). "Fuel-Efficient Gear Shift and Power Split Strategy for Parallel HEVs Based on Heuristic Dynamic Programming and Neural Networks". In: IEEE Transactions on Vehicular Technology 68.10, pp. 95199528. DOI: 10.1109/TVT.2019.2927751.

Lian, Renzong, Jiankun Peng, Yuankai Wu, Huachun Tan, and Hailong Zhang (2020). "Rule-interposing deep reinforcement learning based energy management strategy for power-split hybrid electric vehicle". In: Energy 197, p. 117297.

Liu, Li, Chaosheng Huang, Bingwu Lu, Shuming Shi, Yan Zhang, and Jingmin Cheng (2012). "Study on the design method of time-variant driving cycles for EV based on Markov Process". In: 2012 IEEE Vehicle Power and Propulsion Conference. IEEE, pp. 1277-1281.

Liu, Teng, Xiaosong Hu, Shengbo Eben Li, and Dongpu Cao (2017). "Reinforcement learning optimized look-ahead energy management of a parallel hybrid electric vehicle". In: IEEE/ASME Transactions on Mechatronics 22.4, pp. 1497-1507.

Liu, Teng, Bo Wang, and Chenglang Yang (2018). "Online Markov Chain-based energy management for a hybrid tracked vehicle with speedy Q-learning”. In: Energy 160, pp. 544-555.

Luján, Jose Manuel, Carlos Guardiola, Benjamín Pla, and Alberto Reig (2018). "Analytical optimal solution to the energy management problem in series hybrid electric vehicles”. In: IEEE Transactions on Vehicular Technology 67.8, pp. 68036813.

Miro-Padovani, Thomas, Guillaume Colin, Ahmed Ketfi-Chérif, and Yann Chamaillard (2016). "Implementation of an Energy Management Strategy for Hybrid Electric Vehicles Including Drivability Constraints”. In: IEEE Transactions on Vehicular Technology 65.8, pp. 5918-5929. DOI: 10.1109/TVT.2015.2476820.

Mohebbi, M, M Charkhgard, and M Farrokhi (2005). "Optimal neuro-fuzzy control of parallel hybrid electric vehicles". In: 2005 IEEE Vehicle Power and Propulsion Conference. IEEE, pp. 26-30.

Moser, Dominik, Harald Waschl, Roman Schmied, Hajrudin Efendic, and Luigi del $\operatorname{Re}(2015)$. "Short term prediction of a vehicle's velocity trajectory using its". In: SAE International Journal of Passenger Cars-Electronic and Electrical Systems 8.2015-01-0295, pp. 364-370.

Murphey, Yi L, ZhiHang Chen, Leo Kiliaris, Jungme Park, Ming Kuang, Abul Masrur, and Anthony Phillips (2008). "Neural learning of driving environment prediction for vehicle power management". In: 2008 IEEE International Joint Conference on 
Neural Networks (IEEE World Congress on Computational Intelligence). IEEE, pp. 3755-3761.

Musardo, Cristian, Giorgio Rizzoni, Yann Guezennec, and Benedetto Staccia (2005). "A-ECMS: An adaptive algorithm for hybrid electric vehicle energy management". In: European Journal of Control 11.4-5, pp. 509-524.

Ngo, Viet, Theo Hofman, Maarten Steinbuch, and Alex Serrarens (2012). "Optimal Control of the Gearshift Command for Hybrid Electric Vehicles". In: IEEE Transactions on Vehicular Technology 61.8, pp. 3531-3543. DOI: $10.1109 /$ TVT.2012.2207922.

Opila, Daniel F, Xiaoyong Wang, Ryan McGee, and JW Grizzle (2013). "Real-time implementation and hardware testing of a hybrid vehicle energy management controller based on stochastic dynamic programming". In: Journal of dynamic systems, measurement, and control 135.2, p. 021002.

Opila, Daniel F., Xiaoyong Wang, Ryan McGee, R. Brent Gillespie, Jeffrey A. Cook, and Jessy W. Grizzle (2012). "An Energy Management Controller to Optimally Trade Off Fuel Economy and Drivability for Hybrid Vehicles". In: IEEE Transactions on Control Systems Technology 20.6, pp. 1490-1505. DOI: 10.1109/TCST.2011.2168820.

Paganelli, Gino, Sebastien Delprat, Thierry-Marie Guerra, Janette Rimaux, and JeanJacques Santin (2002). "Equivalent consumption minimization strategy for parallel hybrid powertrains". In: Vehicular Technology Conference. IEEE 55th Vehicular Technology Conference. VTC Spring 2002 (Cat. No. 02CH37367). Vol. 4. IEEE, pp. 2076-2081.

Panday, Aishwarya and Hari Om Bansal (2016). "Energy management strategy for hybrid electric vehicles using genetic algorithm". In: Journal of Renewable and Sustainable Energy 8.1, p. 015701.

Peng, Jiankun, Hongwen He, and Rui Xiong (2017). "Rule based energy management strategy for a series-parallel plug-in hybrid electric bus optimized by dynamic programming". In: Applied Energy 185, pp. 1633-1643.

Qi, Xuewei, Yadan Luo, Guoyuan Wu, Kanok Boriboonsomsin, and Matthew J Barth (2017). "Deep reinforcement learning-based vehicle energy efficiency autonomous learning system". In: 2017 IEEE Intelligent Vehicles Symposium (IV). IEEE, pp. 1228-1233.

Qi, Xuewei, Guoyuan Wu, Kanok Boriboonsomsin, Matthew J Barth, and Jeffrey Gonder (2016). "Data-driven reinforcement learning-based real-time energy management system for plug-in hybrid electric vehicles". In: Transportation Research Record 2572.1, pp. 1-8.

Rawlings, James Blake, David Q Mayne, and Moritz Diehl (2017). Model predictive control: theory, computation, and design. Vol. 2. Nob Hill Publishing Madison, WI.

Schouten, Niels J, Mutasim A Salman, and Naim A Kheir (2002). "Fuzzy logic control for parallel hybrid vehicles". In: IEEE transactions on control systems technology 10.3, pp. 460-468. 
Serrao, Lorenzo, Simona Onori, and Giorgio Rizzoni (2011). “A comparative analysis of energy management strategies for hybrid electric vehicles”. In: Journal of Dynamic Systems, Measurement, and Control 133.3. 031012.

Shafikhani, Iman and Jan Åslund (2021a). "Analytical Solution to Equivalent Consumption Minimization Strategy for Series Hybrid Electric Vehicles". In: IEEE Transactions on Vehicular Technology 70.3, pp. 2124-2137. URL: https : / / doi.org/10.1109/TVT.2021.3059205.

- (2021b). "Energy Management of Hybrid Electric Vehicles with Battery Aging Considerations: Wheel Loader Case Study". In: Control Engineering Practice 110, p. 104759. URL: https: / / doi .org/ 10 . 1016 / j . conengprac . 2021.104759.

Shafikhani, Iman, Erik Frisk, Jan Åslund, and Christofer Sundström (2021). "Realtime Predictive Energy Management Strategies for Series Hybrid Electric Vehicles". In: Manuscript.

Shafikhani, Iman, Christofer Sundström, Jan Åslund, and Erik Frisk (2021). "MPCbased Energy Management System Design for a Series HEV with Battery Life Optimization". In: European Control Conference.

Sotoudeh, Seyedeh Mahsa and Baisravan HomChaudhuri (2020). "A Robust MPCbased Hierarchical Control Strategy for Energy Management of Hybrid Electric Vehicles in Presence of Uncertainty". In: 2020 American Control Conference (ACC). IEEE, pp. 3065-3070.

Sun, Chao, Xiaosong Hu, Scott J Moura, and Fengchun Sun (2014a). "Comparison of velocity forecasting strategies for predictive control in HEVs". In: Dynamic Systems and Control Conference. Vol. 46193. American Society of Mechanical Engineers, V002T20A003.

- (2014b). "Velocity predictors for predictive energy management in hybrid electric vehicles”. In: IEEE Transactions on Control Systems Technology 23.3, pp. 11971204.

Sun, Chao, Fengchun Sun, Xiaosong Hu, J Karl Hedrick, and Scott Moura (2015). "Integrating traffic velocity data into predictive energy management of plug-in hybrid electric vehicles". In: 2015 American Control Conference (ACC). IEEE, pp. 3267-3272.

Suri, Girish and Simona Onori (2016). "A control-oriented cycle-life model for hybrid electric vehicle lithium-ion batteries”. In: Energy 96, pp. 644-653.

Tang, Li, Giorgio Rizzoni, and Simona Onori (2015). "Energy management strategy for HEVs including battery life optimization". In: IEEE Transactions on Transportation Electrification 1.3, pp. 211-222.

Tianheng, Feng, Yang Lin, Gu Qing, Hu Yanqing, Yan Ting, and Yan Bin (2015). "A Supervisory Control Strategy for Plug-In Hybrid Electric Vehicles Based on Energy Demand Prediction and Route Preview". In: IEEE Transactions on Vehicular Technology 64.5, pp. 1691-1700. DOI: 10 . 1109 / TVT . 2014. 2336378. 
Tie, Siang Fui and Chee Wei Tan (2013). "A review of energy sources and energy management system in electric vehicles”. In: Renewable and sustainable energy reviews 20 , pp. $82-102$.

Wu, Jian, C-H Zhang, and N-X Cui (2012). "Fuzzy energy management strategy for a hybrid electric vehicle based on driving cycle recognition". In: International journal of automotive technology 13.7, pp. 1159-1167.

Xie, Shaobo, Xiaosong Hu, Zongke Xin, and James Brighton (2019). “Pontryagin's minimum principle based model predictive control of energy management for a plug-in hybrid electric bus". In: Applied energy 236, pp. 893-905.

Yu, Kaijiang, Xiaozhuo Xu, Qing Liang, Zhiguo Hu, Junqi Yang, Yanan Guo, and Hongwei Zhang (2015). "Model predictive control for connected hybrid electric vehicles". In: Mathematical Problems in Engineering 2015.

Yuan, Zou, Liu Teng, Sun Fengchun, and Huei Peng (2013). "Comparative study of dynamic programming and Pontryagin's minimum principle on energy management for a parallel hybrid electric vehicle”. In: Energies 6.4, pp. 2305-2318.

Zeng, Xiangrui and Junmin Wang (2015). “A parallel hybrid electric vehicle energy management strategy using stochastic model predictive control with road grade preview". In: IEEE Transactions on Control Systems Technology 23.6, pp. 24162423.

Zhang, Chen, Ardalan Vahidi, Pierluigi Pisu, Xiaopeng Li, and Keith Tennant (2009). "Role of terrain preview in energy management of hybrid electric vehicles". In: IEEE transactions on Vehicular Technology 59.3, pp. 1139-1147.

Zhang, Fengqi, Lihua Wang, Serdar Coskun, Hui Pang, Yahui Cui, and Junqiang $\mathrm{Xi}$ (2020). "Energy management strategies for hybrid electric vehicles: Review, classification, comparison, and outlook". In: Energies 13.13, p. 3352.

Zhang, Fengqi, Junqiang Xi, and Reza Langari (2017). "Real-Time Energy Management Strategy Based on Velocity Forecasts Using V2V and V2I Communications". In: IEEE Transactions on Intelligent Transportation Systems 18.2, pp. 416-430. DOI: 10.1109 /TITS.2016.2580318.

Zhang, Y (2010). "DIRECT algorithm and driving cycle recognition based optimization study for hybrid electric vehicle". PhD thesis. Ph. D. Thesis, Dept. Mech. Eng., Beijing Inst. of Tech., Beijing, China.

Zhou, Yang, Alexandre Ravey, and Marie-Cécile Péra (2019). “A survey on driving prediction techniques for predictive energy management of plug-in hybrid electric vehicles". In: Journal of Power Sources 412, pp. 480-495.

Zulkefli, Mohd Azrin Mohd, Jianfeng Zheng, Zongxuan Sun, and Henry X Liu (2014). "Hybrid powertrain optimization with trajectory prediction based on inter-vehicle-communication and vehicle-infrastructure-integration". In: Transportation Research Part C: Emerging Technologies 45, pp. 41-63. 
Papers 



\section{Papers}

The papers associated with this thesis have been removed for copyright reasons. For more details about these see:

http://urn.kb.se/resolve?urn=urn:nbn:se:liu:diva-178628 



\section{Dissertations \\ Division of Vehicular Systems \\ Department of Electrical Engineering \\ Linköping University}

No. 1 Magnus Pettersson, Driveline Modeling and Control, 1997.

No. 2 Lars Eriksson, Spark Advance Modeling and Control, 1999.

No. 3 Mattias Nyberg, Model Based Fault Diagnosis: Methods, Theory, and Automotive Engine Applications, 1999.

No. 4 Erik Frisk, Residual Generation for Fault Diagnosis, 2001.

No. 5 Per Andersson, Air Charge Estimation in Turbocharged Spark Ignition Engines, 2005.

No. 6 Mattias Krysander, Design and Analysis of Diagnosis Systems Using Structural Methods, 2006.

No. 7 Jonas Biteus, Fault Isolation in Distributed Embedded Systems, 2007.

No. 8 Ylva Nilsson, Modelling for Fuel Optimal Control of a Variable Compression Engine, 2007.

No. 9 Markus Klein, Single-Zone Cylinder Pressure Modeling and Estimation for Heat Release Analysis of SI Engines, 2007.

No. 10 Anders Fröberg, Efficient Simulation and Optimal Control for Vehicle Propulsion, 2008.

No. 11 Per Öberg, A DAE Formulation for Multi-Zone Thermodynamic Models and its Application to CVCP Engines, 2009.

No. 12 Johan Wahlström, Control of EGR and VGT for Emission Control and Pumping Work Minimization in Diesel Engines, 2009.

No. 13 Anna Pernestål, Probabilistic Fault Diagnosis with Automotive Applications, 2009.

No. 14 Erik Hellström, Look-ahead Control of Heavy Vehicles, 2010.

No. 15 Erik Höckerdal, Model Error Compensation in ODE and DAE Estimators with Automotive Engine Applications, 2011.

No. 16 Carl Svärd, Methods for Automated Design of Fault Detection and Isolation Systems with Automotive Applications, 2012.

No. 17 Oskar Leufvén, Modeling for Control of Centrifugal Compressors, 2013.

No. 18 Christofer Sundström, Model Based Vehicle Level Diagnosis for Hybrid Electric Vehicles, 2014. 
No. 19 Andreas Thomasson, Modeling and control of actuators and co-surge in turbocharged engines, 2014.

No. 20 Emil Larsson, Model Based Diagnosis and Supervision of Industrial Gas Turbines, 2014.

No. 21 Andreas Myklebust, Dry Clutch Modeling, Estimation, and Control, 2014.

No. 22 Tomas Nilsson, Optimal Engine Operation in a Multi-Mode CVT Wheel Loader, 2015.

No. 23 Daniel Jung, Diagnosability Performance Analysis of Models and Fault Detectors, 2015.

No. 24 Martin Sivertsson, Optimal Control of Electrified Powertrains, 2015.

No. 25 Peter Nyberg, Evaluation, Generation, and Transformation of Driving Cycles, 2015.

No. 26 Kristoffer Lundahl, Models and Critical Maneuvers for Road Vehicles, 2016.

No. 27 Vaheed Nezhadali, Modeling and Optimal Control of Heavyduty Powertrains, 2016.

No. 28 Xavier Llamas, Modeling and Control of EGR on Marine TwoStroke Diesel Engines, 2018.

No. 29 Sergii Voronov, Machine Learning for Predictive Maintenance, 2020.

No. 30 Victor Fors, Autonomous Vehicle Maneuvering at the Limit of friction, 2020.

No. 31 Fatemeh Mohseni, Decentralized Optimal Control for Multiple Autonomous Vehicles in Traffic Scenarios, 2021.

No. 32 Mahdi Morsali, Trajectory Planning of an Autonomous Vehicle in Multiple-Vehicle Traffic Scenarios, 2021.

No. 33 Kristoffer Ekberg, Modeling and Optimal Control for Dynamic Driving of Hybridized Vehicles with Turbocharged Diesel Engines, 2021. 


\section{FACULTY OF SCIENCE AND ENGINEERING}

Linköping Studies in Science and Technology, Dissertation No. 2165, 2021

Department of Electrical Engineering

Linköping University

SE-581 83 Linköping, Sweden

www.liu.se 